\title{
Single-Shot Three-Dimensional Orientation Imaging of Nanorods Using Spin to Orbital Angular Momentum Conversion
}

\author{
Tomás Fordey, Petr Bouchal,* Petr Schovánek, Michal Baránek, Zdeněk Bouchal, Petr Dvorák, \\ Martin Hrtoň, Katarína Rovenská, Filip Ligmajer, Radim Chmelík, and Tomáš Sikola
}

Cite This: Nano Lett. 2021, 21, 7244-7251

Read Online

\section{ACCESS | Lill Metrics \& More | 回 Article Recommendations ｜（） Supporting Information}

ABSTRACT: The key information about any nanoscale system relates to the orientations and conformations of its parts. Unfortunately, these details are often hidden below the diffraction limit, and elaborate techniques must be used to optically probe them. Here we present imaging of the 3D rotation motion of metal nanorods, restoring the distinct nanorod orientations in the full extent of azimuthal and polar angles. The nanorods imprint their $3 \mathrm{D}$ orientation onto the geometric phase and space-variant polarization of the light they scatter. We manipulate the light angular momentum and generate optical vortices that create self-interference images providing the nanorods' angles via digital
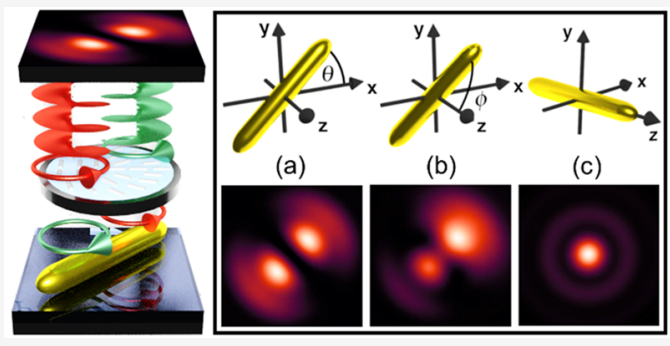
processing. After calibration by scanning electron microscopy, we demonstrated time-resolved 3D orientation imaging of sub-100 nm nanorods under Brownian motion (frame rate up to 500 fps). We also succeeded in imaging nanorods as nanoprobes in live-cell imaging and reconstructed their 3D rotational movement during interaction with the cell membrane (100 fps).

KEYWORDS: Nanorods, Orientation imaging, Dark-field microscopy, Light angular momentum, Optical vortices, Space-variant polarization

\section{INTRODUCTION}

Nanoparticles made of noble metals exhibit remarkable shapedependent optical properties associated with surface plasmon resonance (SPR) excitation. ${ }^{1,2}$ Novel applications using nanoparticles as contrast agents and imaging and sensing probes have emerged thanks to their biocompatibility, ability to enhance the driving field without bleaching, and SPR sensitivity to the nanoparticle size and its surrounding environment. ${ }^{3,4}$ In recent years, elongated nanoparticles (nanorods) exhibiting longitudinal SPR (LSPR) have attracted a growing interest. The nanorods became popular thanks to weak radiation damping due to small volumes, high lightscattering efficiencies, and large local-field enhancement factors $^{5}$ and the possibility of manipulating their aspect ratio. ${ }^{6}$ Thanks to their shape anisotropy, nanorods were also deployed as sensitive orientation sensors in the conformation and dynamics studies of biological systems. ${ }^{7}$ However, orientation imaging can go beyond biophotonic applications and can be used as a powerful tool for the study of nanorod supercrystals, ${ }^{8}$ superlattices, ${ }^{9}$ or nanorod chains ${ }^{10}$ deployed in new plasmonic-based nanoimaging devices. ${ }^{11}$ Originally, the problem of orientation imaging was restricted only to $2 \mathrm{D}$ measurements (i.e., in-plane rotation) and addressed by different modifications of dark-field polarization microscopy, ${ }^{12-16}$ polarization-sensitive photothermal imaging, ${ }^{7}$ or confocal microscopy combined with higher order laser modes. ${ }^{17}$ In the dark-field microscopy methods, the orientation measurement had to be realized using successive images recorded with different polarization directions of illumination, ${ }^{12-14}$ otherwise the orientation ambiguity arose. ${ }^{15}$ In the confocal microscopy approach to orientation imaging, scanning by a doughnut laser beam was needed. This technique prevents imaging with high temporal resolution, which is critical for most applications. Further progress in orientation imaging was achieved by attempting a 3D orientation measurement using defocused dark-field microscopy $^{18-20}$ and differential interference contrast (DIC) microscopy. ${ }^{21,22}$ It was demonstrated that defocused darkfield images encode 3D orientation; however, large defocusing $(\sim 1 \mu \mathrm{m})$ with an immersion microscope objective (numerical aperture 1.3) is needed. ${ }^{20}$ Defocusing is necessary even to measure pure in-plane rotation, and it results in a decrease in the spatial resolution and image spreading, which is limited under the low-light conditions occurring in most practical applications. Using DIC, the measurement of the in-plane rotation combined with some elevation of the nanorod (out-ofplane rotation) was demonstrated and even supplemented by

Received: June 11, 2021

Revised: August 9, 2021

Published: August 26, 2021 
position tracking in the later work. ${ }^{23,24}$ However, the out-ofplane rotation was measured only for small nanorod elevations (up to $45^{\circ}$ ) because larger elevation angles prevented the correct evaluation of DIC images. ${ }^{23}$ The impossibility of distinguishing the nanorod's rotational motion from its axial movement required deploying parallax microscopy and a feedback loop algorithm to control the focal plane position. ${ }^{24}$ Hence, simple, fast, and reliable $3 \mathrm{D}$ orientation imaging in the full extent of in-plane and out-of-plane rotations has remained a challenge.

In this Letter, we capitalize on the recent progress in (nano)photonics that has brought new types of light fields with a spatially structured amplitude, ${ }^{25}$ phase, $^{26}$ or polarization. ${ }^{27,28}$ We demonstrate a new strategy for the single-shot 3D orientation imaging of metal nanorods that is built on thoughtful manipulations with the light's angular momentum. The nanorods imprint their 3D orientation onto the geometric (Pancharatnam-Berry) phase and the space-variant polarization of the scattered light arising from the excited LSPR. To measure the nanorod azimuthal and polar angles, we manipulate the spin and orbital angular momenta (SAM and OAM) of the scattered light and perform mutual conversion between them using a Q-plate, ${ }^{29-31}$ a space-variant anisotropic element provided by fourth-generation technology. ${ }^{32,33}$ In this way, optical vortices are generated, whose self-interference images provide information on the $3 \mathrm{D}$ nanorod orientation. Images encode the in-plane orientation of the nanorod without the ambiguity of the azimuthal angles previously reported. ${ }^{15}$ Sensing the out-of-plane rotation requires minimal nanorod defocusing $(\sim 100 \mathrm{~nm}$ with an immersion microscope objective of numerical aperture 1.0). This is just a small fraction of the defocusing required in previous studies, and the nanorods are safely kept within the depth of field during the imaging. On top of that, the measurement takes full advantage of the vortex image pattern (double-helix point spread function (DH PSF)) that enhances the Fisher information. ${ }^{34}$ The nanorods are excited by spatially incoherent light; hence, the recorded images are free from the unwanted coherent artifacts. Measurements are performed in a wide-field mode, meaning that a single image provides complete information on the lateral positions and 3D orientations of all nanorods in the field of view. Although the axial positions of nanorods are unavailable in current experiments, the proposed method can be combined with localization techniques introduced in a separate channel of the microscope. The optical performance of our method is demonstrated by the time-resolved 3D orientation imaging of colloidal sub-100 nm nanorods under Brownian motion and the monitoring of the rotational motion of nanoprobes bound to living cells. The tracking of nanorods and the monitoring of their $3 \mathrm{D}$ rotational motion are possible with high temporal resolution (frame rate up to $500 \mathrm{fps}$ ). This opens new possibilities for studying very fast events such as the conformational dynamics of biological cells, ${ }^{35}$ the rotation of biological motor proteins, ${ }^{36}$ or nanorod motors. ${ }^{37}$

\section{RESULTS}

Experimental Strategy. Thanks to its anisotropic shape, a nanorod can support two SPR modes corresponding to the transverse SPR and LSPR, respectively. The resonant wavelengths of both modes are spectrally separated and thus can be independently excited. The developed orientation imaging can gain information from both transverse SPR and LSPR, but here we concentrate on high-aspect-ratio nanorods in which LSPR dominates. The nanorod is considered as a dipolar light source with the electric dipole moment (DM) oscillating along the nanorod's orientation described by the azimuthal and polar angles, $\theta$ and $\phi$. The information on the $3 \mathrm{D}$ nanorod orientation is thus encoded in the spatial and polarization structures of the emitted radiation, varying with $\theta$ and $\phi$. Our theoretical model builds on two imaging modes related to the specific nanorod orientations: the lateral imaging mode (LIM), applicable when the nanorod lies in the plane perpendicular to the optical axis $(\phi=\pi / 2)$, and the axial imaging mode (AIM), applicable when it lies along the optical axis $(\phi=0)$. The intensity spots detected in the LIM and AIM correspond to the DH PSF and to the Airy disk, respectively (top and bottom optical paths in Figure 1). When imaging a nanorod with an

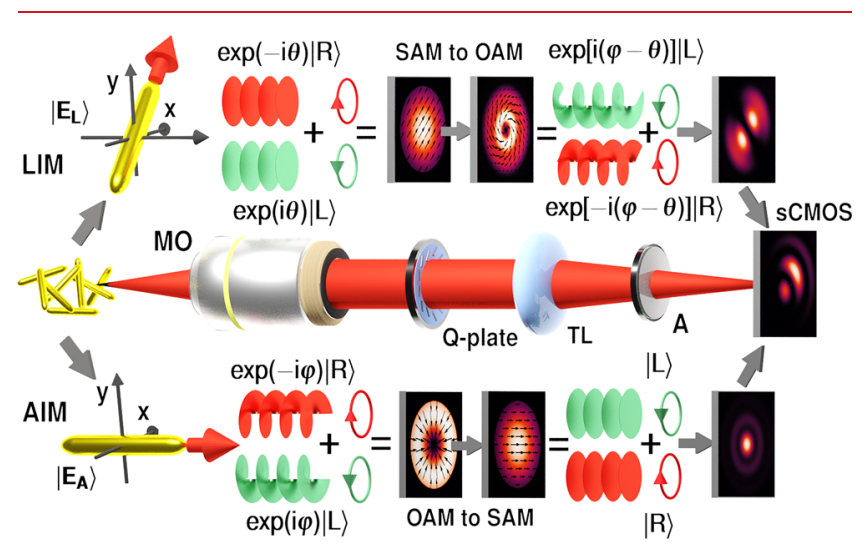

Figure 1. Principle of orientation imaging of nanorods considered as electric dipoles. Information on an arbitrary $3 \mathrm{D}$ nanorod orientation is carried through the LIM and AIM using light originating from the lateral and axial projection of the oscillating electric DM. In the LIM and AIM, the light undergoes SAM to OAM and reverse OAM to SAM conversion as it passes through the same optical system composed of a microscope objective (MO), a Q-plate, a tube lens (TL), an analyzer (A), and an sCMOS detector. The 3D nanorod orientation is determined by processing a slightly defocused image whose generation we explain by the self-interference of subimages formed in the LIM and AIM.

arbitrary 3D orientation, we can always decompose its 3D DM oscillation into the LIM and AIM components. The LIM and AIM light fields are polarization-resolved and undergo different transformations even though they pass through the same optical system. Polarization projection by an analyzer lets them interfere and create the resulting image (the sCMOS (scientific complementary metal-oxide-semiconductor) image in Figure 1). We will explain this process in greater detail as follows, as we will follow the state vectors $\left|E_{\mathrm{L}}\right\rangle$ and $\left|E_{\mathrm{A}}\right\rangle$ related to the LIM and AIM on their path through the optical system consisting of a microscope objective (MO), a Q-plate, a tube lens (TL), an analyzer (A), and an sCMOS detector. After any transformation made, we obtain the resulting field as $|E\rangle=w_{\mathrm{L}} \mid$ $\left.E_{\mathrm{L}}\right\rangle+w_{\mathrm{A}}\left|E_{\mathrm{A}}\right\rangle$, where the used weights depend on the polar angle $\phi, w_{\mathrm{L}}=\sin \phi$ and $w_{\mathrm{A}}=\cos \phi$.

First, let us consider light in the LIM, which is generated by the projection of the nanorod's electric DM oscillation into the $x, y$ plane $(\phi=\pi / 2)$. This scattered light has linear polarization $|\theta\rangle=|x\rangle \cos \theta+|y\rangle \sin \theta$ following the nanorod azimuthal direction (LIM path in Figure 1). The linear polarization can be decomposed into left- and right-handed circular polarizations, $|L\rangle$ and $|R\rangle$, carrying opposite SAM given by $\pm \hbar$ per photon. ( $\hbar$ is the reduced Planck constant). When the light 

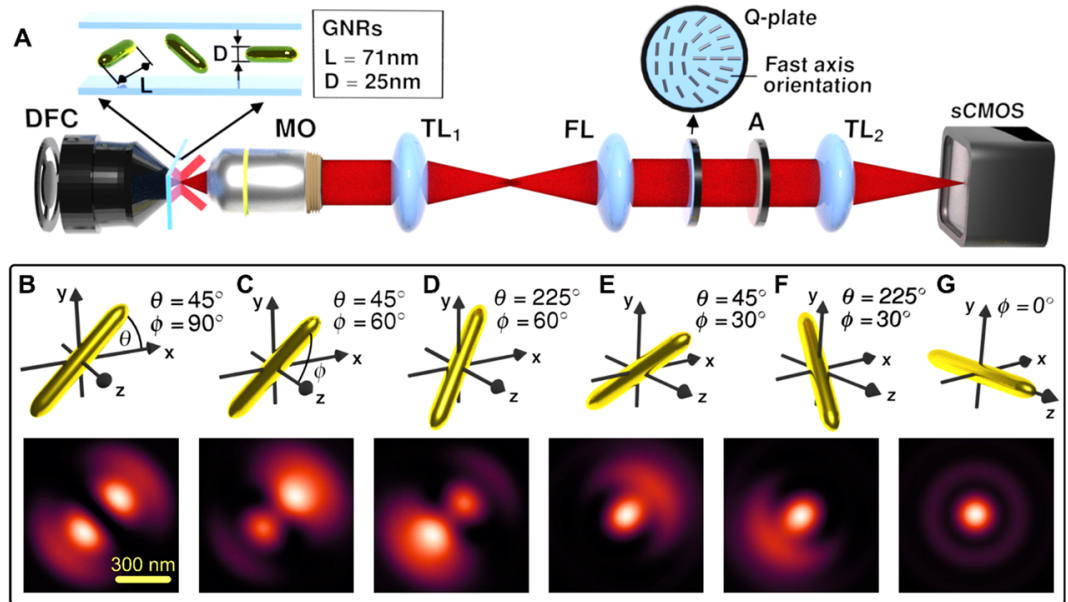

Figure 2. Single-shot 3D orientation imaging of metal nanorods. (A) Experimental setup: dark-filed condenser (DFC), microscope objective (MO), tube lenses $\left(\mathrm{TL}_{1}\right.$ and $\left.\mathrm{TL}_{2}\right)$, Fourier lens $(\mathrm{FL})$, Q-plate, analyzer $(\mathrm{A})$, and sCMOS camera. $(\mathrm{B}-\mathrm{G})$ Calculated image patterns providing information on the $3 \mathrm{D}$ nanorod orientation specified by the azimuthal and polar angles, $\theta$ and $\phi$.

from the lateral DM projection given by the angles $\theta$ and $\phi=$ $\pi / 2$ is expressed in the used vector basis, the phase shifts $\pm \theta$ are imposed on the $|L\rangle$ and $|R\rangle$ states. ${ }^{32,33}$ This means that the geometric-phase difference $2 \theta$ is introduced between the circular polarizations (Figure 1). If a Q-plate ${ }^{31}$ is placed into the optical path, then the light undergoes SAM to OAM conversion, in which the reversion of the circular polarizations is accompanied by the generation of optical vortex beams. The vortex beams are characterized by helical wavefronts, ${ }^{38-40}$ meaning the light is twisted around the optical axis, and each of its photons carries OAM of $l \hbar$. The integer $l$ is called the topological charge, and it specifies how many twists the wavefront makes per one wavelength. In our experiment, the light with polarization $|L\rangle(+\hbar$ SAM $)$ is transformed to a vortex beam with polarization $|R\rangle(-\hbar$ SAM) and the helical wavefront with topological charge $l=1(+\hbar$ OAM $)$. An analogous operation is simultaneously carried out on the $|R\rangle$ input state, and hence a pair of vortices with $l= \pm 1$ and opposite circular polarizations is generated from the linearly polarized light in the LIM. The resulting field created by the superposition of circularly polarized components modified by the Q-plate can be written as $\left|E_{\mathrm{L}}\right\rangle \propto \mathrm{e}^{i(\varphi-\theta)}|L\rangle+\mathrm{e}^{-i(\varphi-\theta)}|R\rangle$. After shaping with the tube lens (TL), the state vector of this field represents a space-variant polarization $\left|E_{\mathrm{L}}\right\rangle=-i A_{\mathrm{L}}(r) \mid \varphi-$ $\theta\rangle$, where $A_{\mathrm{L}}$ is the amplitude obtained by solving the diffraction integral, and $r$ and $\varphi$ are the cylindrical coordinates (see the Supporting Information). This is a linear polarization whose electric field oscillates in the direction determined by the angle $\varphi-\theta$ at points with the azimuthal coordinate $\varphi, \mid \varphi$ $-\theta\rangle=|x\rangle \cos (\varphi-\theta)+|y\rangle \sin (\varphi-\theta)$. Because the oscillation direction is ambiguous at the beam center, the amplitude is zero here, and an annular intensity spot given by $\left|A_{\mathrm{L}}\right|^{2}$ arises. By the polarization projection using an analyzer (A), two lobes are created from the annular spot. When the analyzer direction coincides with the reference direction of the Q-plate (here chosen as the $x$ axis), the centers of gravity of the lobes determine the azimuthal orientation of the nanorod, $\theta$. The intensity pattern is then given by $I_{\mathrm{L}}=\left|A_{\mathrm{L}}(r)\right|^{2} \cos ^{2}(\varphi-\theta)$ (LIM path in Figure 1).

The second component of the scattered light field comes from the axial projection of the nanorod's DM oscillation (AIM). The collected light has a space-variant polarization with the state vector $\left|E_{\mathrm{A}}\right\rangle \propto|\varphi\rangle$, which is known as the radial polarization. ${ }^{41}$ The electric field is linearly polarized at each point of the lateral plane and oscillates along the radial direction $|\varphi\rangle=|x\rangle \cos \varphi+|y\rangle \sin \varphi$. The beam intensity creates an annular spot at the back focal plane of the microscope objective. The vortex helical phases with the topological charge $l= \pm 1$ are imposed on the $|R\rangle$ and $|L\rangle$ states to represent the radial polarization in the circular polarization basis, $\left|E_{\mathrm{A}}\right\rangle \propto \mathrm{e}^{i \varphi}|L\rangle+\mathrm{e}^{(-i \varphi)}|R\rangle$ (AIM path in Figure 1). The radial polarization of the resulting field becomes the horizontal linear polarization behind the Q-plate, $\left|E_{\mathrm{A}}\right\rangle \propto|L\rangle+|R\rangle$. The light shaped by the tube lens creates a bright spot with the intensity determined as $I_{\mathrm{A}}=\left|A_{\mathrm{A}}(r)\right|^{2}$, where $A_{\mathrm{A}}$ is the amplitude of the Airy disk.

The image intensity $I_{\mathrm{L}}$ or $I_{\mathrm{A}}$ is detected only when the nanorod is positioned exactly in a plane perpendicular to the optical axis $(\phi=\pi / 2)$ or aims along the optical axis $(\phi=0)$, respectively. The $I_{\mathrm{L}}$ image comprises two lobes of equal intensities (known in another context as a $\mathrm{DH} \mathrm{PSF}^{42}$ ), uniquely determining the nanorod azimuthal orientation, $\theta$ (Figure 2B). The fully out-of-plane orientation of the nanorod, on the contrary, then manifests itself as the Airy diffraction pattern, $I_{\mathrm{A}}$ (Figure $2 \mathrm{G}$ ). When the nanorod has a general $3 \mathrm{D}$ orientation with arbitrary angles $\theta$ and $\phi$, the fundamental electric fields of the LIM and AIM are projected into same direction by the analyzer, and the resulting image is created by their coherent superposition. The weighting coefficients belonging to the LIM and AIM depend on the polar angle and are given by $w_{\underline{L}}=\sin \phi$ and $w_{\mathrm{A}}=\cos \phi$, respectively. Detecting a slightly defocused image, the amplitudes $A_{\mathrm{L}}$ and $A_{\mathrm{A}}$ become complex, and the image intensity is given by $I=I_{\mathrm{L}}+I_{\mathrm{A}}$ $+I_{\mathrm{LA}}$, where the interference term can be written as $I_{\mathrm{LA}} \propto i$ sin $2 \phi \cos (\varphi-\theta)\left(A_{\mathrm{A}} A_{\mathrm{L}}^{*}-A_{\mathrm{L}} A_{\mathrm{A}}^{*}\right)$ (sCMOS image spot in Figures 1 and $2 \mathrm{C}-\mathrm{F})$. The interference redistributes the overall intensity between the two LIM lobes and the central AIM spot depending on the polar angle, $\phi$. The polar angle sign exchanges constructive and destructive interference in the lobes, and hence we measure the polar angle without ambiguity (cf. Figure 2C with Figure 2D and Figure 2E with Figure 2F). The azimuthal angle $\theta$ of the nanorod follows from the angular orientation of the image spot. Examples of images uniquely encoding the $3 \mathrm{D}$ orientation of nanorods are presented in 

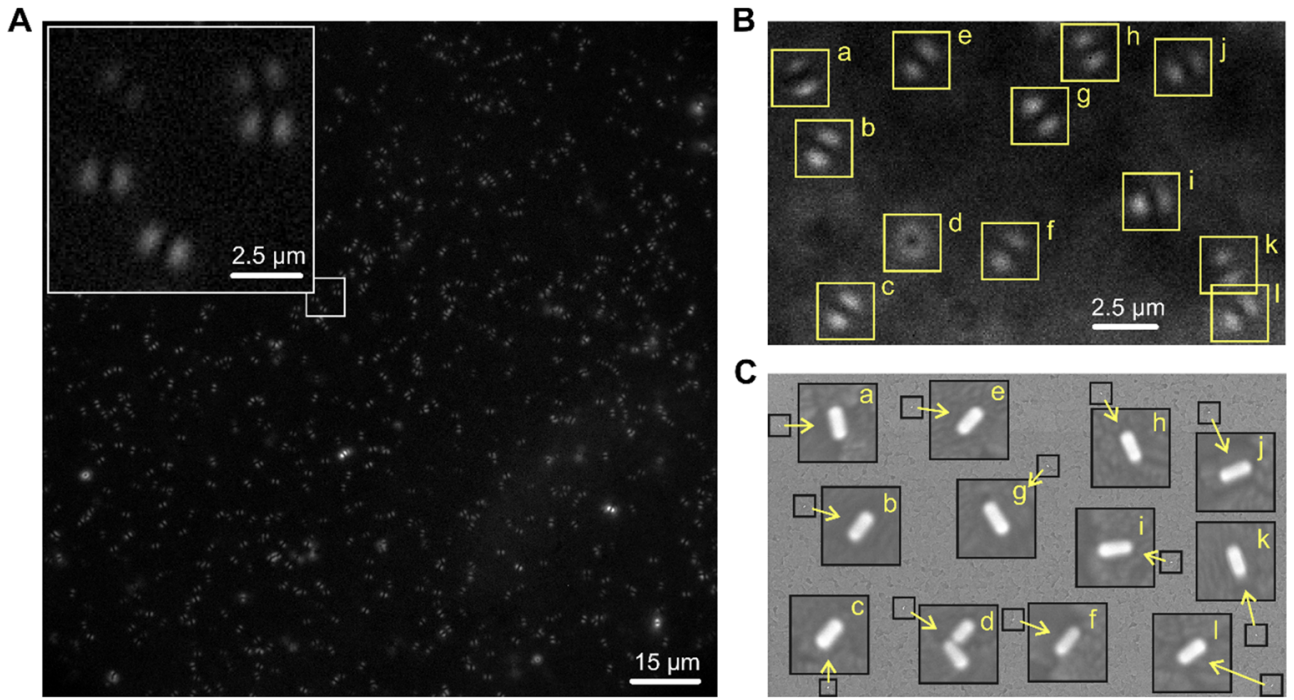

Figure 3. Imaging of a calibration sample and evaluation of the measurement accuracy. (A) Measurement of the in-plane rotation of randomly distributed sub-100 nm colloidal gold nanorods on an ITO-coated glass substrate using the proposed method. (B) Close-up image comprising 12 nanorods. (C) Corresponding reference SEM image of the same field of view as in panel B.
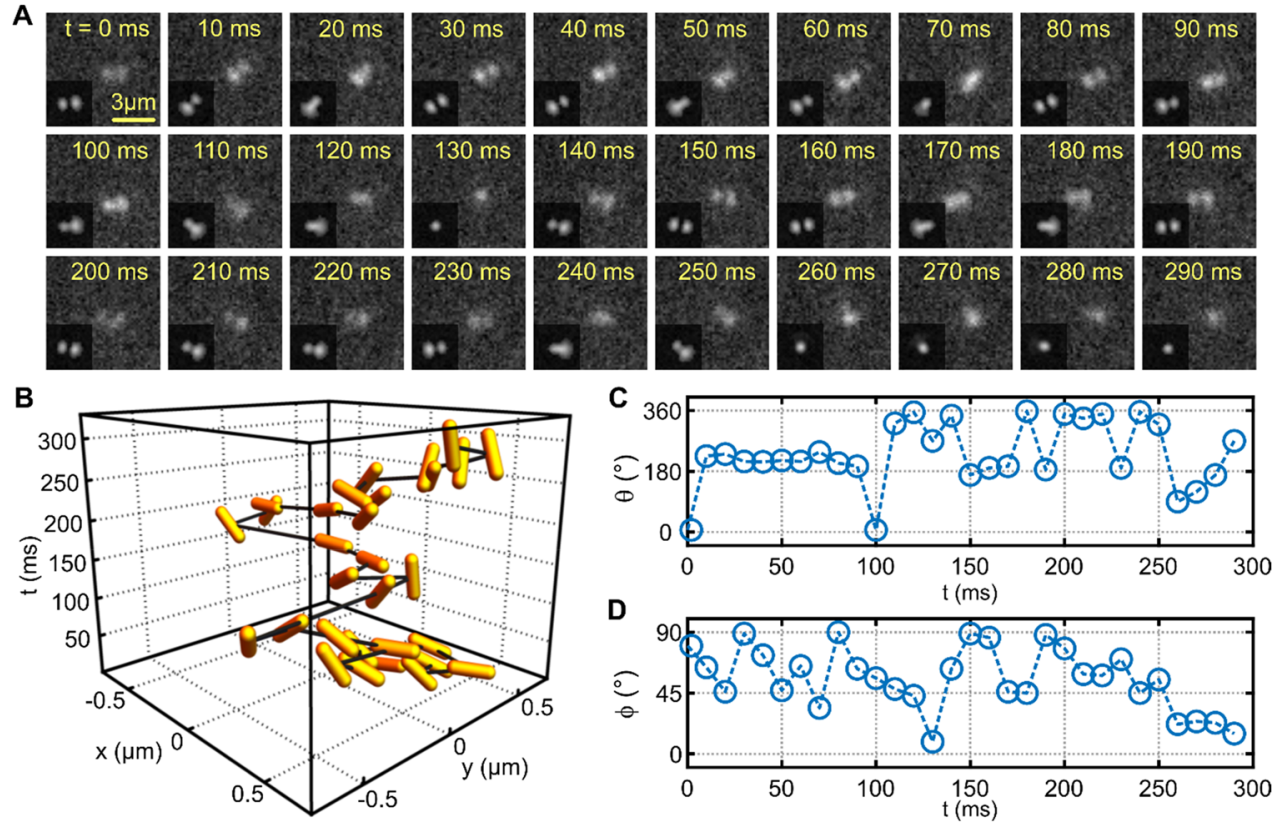

Figure 4. 3D orientation imaging of nanorods moving by Brownian motion. (A) Gradual variations in the azimuthal and polar angles $\theta$ and $\phi$ of a single nanorod are reflected in variations in the detected image patterns taken with a time step of $10 \mathrm{~ms}$. Insets are theoretical images with the best compliance with experimental images. (B) Reconstruction of the single nanorod 3D orientation during its Brownian motion. Orientation is extracted from the measurement presented in panel A. (C) Temporal evolution of the nanorod azimuthal angle, $\theta$. (D) Temporal evolution of the nanorod polar angle, $\phi$.

Figure $2 \mathrm{~B}-\mathrm{G}$. The obtained results were checked by nanophotonic boundary element method (BEM) simulation software (Supporting Information).

Experimental Setup. Experimental results were obtained using the optical system schematically shown in Figure 2. The setup is designed as a dark-field transmission microscope equipped with an add-on imaging module. Illumination by quasi-monochromatic spatially incoherent light with random polarization was provided by a high-power LED (Thorlabs SOLIS-623C, $623 \mathrm{~nm}$, full width at half-maximum $50 \mathrm{~nm}$ ). To excite the LSPR in nanorods of an arbitrary $3 \mathrm{D}$ orientation, we used an oil immersion dark-field condenser (DFC) (NA =
1.4), and hence plane waves with wave vectors forming a hollow cone illuminated the sample. The light scattered by the nanorods was collected by a water dipping microscope objective (MO) (Nikon $60 \times, \mathrm{NA}=1$ ) and subsequently transformed by a tube lens $\left(\mathrm{TL}_{1}\right)$ (Nikon, $f=200 \mathrm{~mm}$ ), creating a magnified dark-field image of nanorods at the output port of the microscope. The add-on imaging module then consisted of a Fourier lens (FL) (Nikon camera lens, $f=50$ $\mathrm{mm}$ ), a Q-plate (Thorlabs WPV10L-633), an analyzer (A), a second tube lens $\left(\mathrm{TL}_{2}\right)$ (Nikon camera lens, $f=80 \mathrm{~mm}$ ), and an sCMOS camera (Andor SONA). Because the lenses $\mathrm{TL}_{1}$ and FL form a $4 \mathrm{~F}$ system, projecting the back focal plane of the 

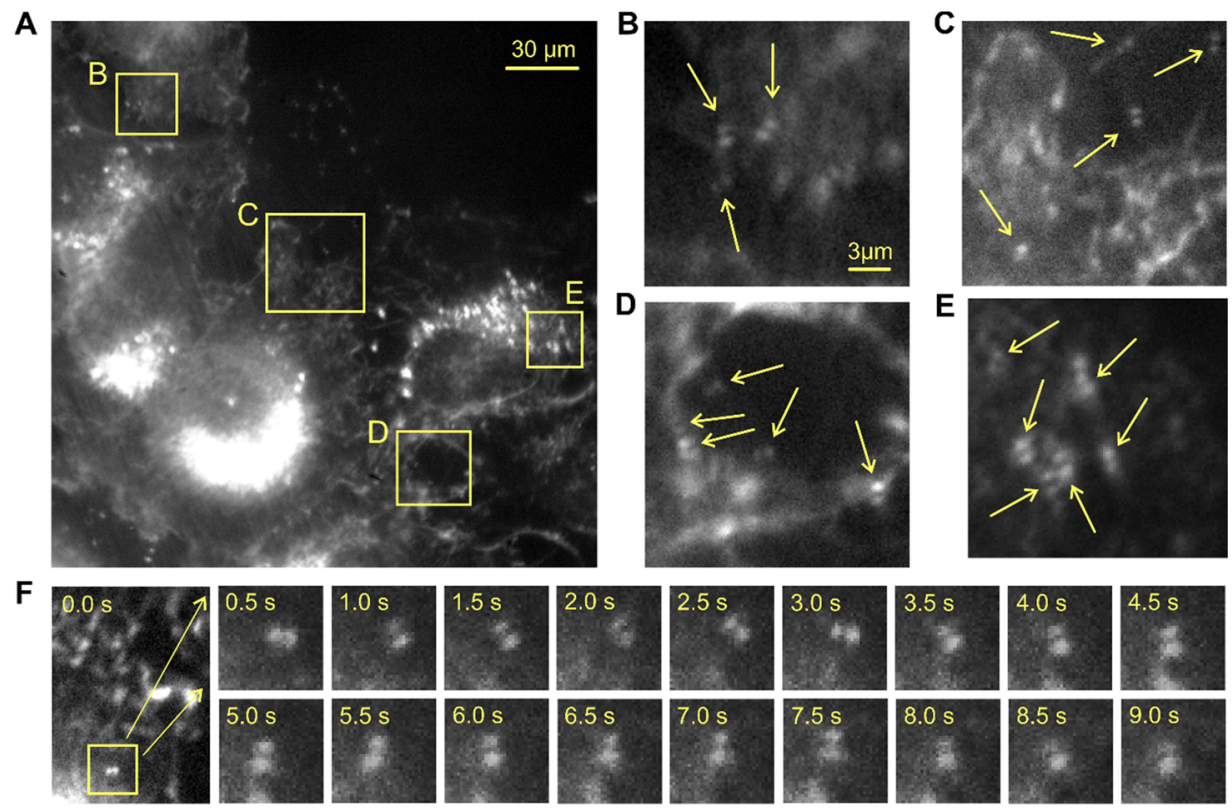

G

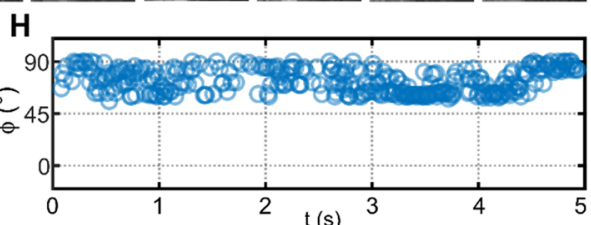

Figure 5. Orientation imaging of gold nanorods in live-cell imaging. (A) Typical image of BLM melanoma cells with added colloidal gold nanorods. (B-E) Enlarged images of the areas marked in panel A with arrows showing detailed images of the present nanorods. (F) Time-resolved images of nanorod binding to the cell membrane. $(\mathrm{G})$ Temporal evolution of the azimuthal angle $\theta$ during the interaction of the nanorod with the cell membrane. $(\mathrm{H})$ Temporal evolution of the polar angle $\phi$ during the interaction of the nanorod with the cell membrane.

MO to the Q-plate, the operation principle is the same as that for the simplified arrangement in Figure 1.

Calibration Measurement. The developed technique was first experimentally tested in a simplified configuration by measuring only the in-plane nanorod orientation (LIM, $\phi=\pi / 2$ ) because calibration samples were easily available, allowing one to assess the method's accuracy. We used a commercial nanorod suspension (Nanopartz A12-25-650, diameter $25 \mathrm{~nm}$, length $71 \mathrm{~nm}$ ) that was dropcasted onto a glass substrate covered by a thin layer of indium tin oxide, allowed to fully dry out, and subsequently imaged using our method. The resulting image showing the full field of view is provided in Figure 3A. The deposited nanorods naturally lay flat on the substrate $(\phi \approx \pi / 2)$ and are thus imaged as $\mathrm{DH}$ PSFs. The orientation of the DH PSF lobes then reveals the inplane azimuthal orientation $\theta$ of the individual nanorods. This was verified using reference imaging in a scanning electron microscope (SEM). By comparing the orientation imaging of the nanorods (Figure $3 \mathrm{~B}$ ) with the reference SEM image of the same area (Figure 3C), a good visual compliance is apparent. To assess these results quantitatively, we prepared an imageprocessing algorithm (see Supporting Information), allowing us to extract the angular orientation of individual DH PSFs in the whole field of view and compare them with the corresponding SEM images. According to this test, we estimate the accuracy of our method by a standard deviation of $2.5^{\circ}$, a remarkable value in the context of orientation imaging (see the Supporting Information). We also paid attention to an Lshaped cluster of nanorods (area " $\mathrm{d}$ " in Figure 3C), which resulted in an annular image spot (area "d") in Figure 3B). The light originating from LSPRs excited in the two perpendicular nanorods produced two DH PSFs with mutually perpendicular lobes. By an incoherent sum of these lobes, an almost perfectly symmetrical ring appeared in the detected image. The optical image of L-shaped nanorods clearly distinguished from single nanorods shows that the method is applicable beyond the present study and can also be promising for the probing of more complex nanostructures such as nanorod clusters ${ }^{43}$ and V-shaped ${ }^{44}$ or chiral plasmonic nanoantennas. ${ }^{45}$

Orientation Imaging of Gold Nanorods Undergoing Brownian Motion. The practical real-world applicability of the proposed method was demonstrated by orientation imaging of colloidal gold nanorods undergoing Brownian motion in a liquid environment. Nanorods from the commercial suspension (see above) moved freely in a droplet of deionized water while exhibiting fast changes in their 3D orientation. During this motion, the detected image patterns rapidly changed their appearance in line with the theoretical model previously described. We operated an sCMOS camera with a frame rate of $100 \mathrm{~Hz}$ to adequately sample the fast nanorod movement while providing a sufficient signal-to-noise ratio. At the cost of higher background noise, we were also able to record images with frame rates up to $500 \mathrm{~Hz}$ (see the Supporting Information). Raw images mapping the gradual motion of a selected single nanorod are shown in Figure 4A (time step of $10 \mathrm{~ms}$ ). Some image spots in Figure 4A well approach the pure DH PSF (time of 30, 80, and $150 \mathrm{~ms}$ ) or the Airy disk (time of 130 and $290 \mathrm{~ms}$ ), meaning that the nanorod was located almost precisely in the lateral or axial position, resulting in a pure LIM or AIM. At other times, the 
nanorod passed through positions with the polar angle $\phi$ varying between the two extremes $(0$ and $\pi / 2)$, resulting in the mixed scattering patterns. Using the developed imaging model previously described, $\phi$ and $\theta$ can be estimated by comparing the analytically calculated spots to the experimental data. Theoretical spots with the best compliance with experimental images are shown next to raw experimental data in the Figure 4A. Reconstruction results providing angles $\theta$ and $\phi$ are plotted in Figure 4B-D. Figure 4B shows an $x, y$-plane trajectory of a selected nanorod together with its 3D orientation estimate at each point (the $z$ axis is time). Figure $4 \mathrm{C}, \mathrm{D}$ reveals the temporal changes of the azimuthal angle, $\theta$, and the polar angle, $\phi$, respectively. The results presented in Figure 4 are a substack of the data set containing 350 images taken during the course of $3.5 \mathrm{~s}$. Reconstruction of the whole data set including the lateral displacement of the nanorod is demonstrated in the Supporting Information. Reconstruction of the nanorod orientation, involving neural network denoising and best correlation fitting, was fully automated and is also described in the Supporting Information.

An evaluation of the reconstruction procedure and accuracy of $3 \mathrm{D}$ orientation imaging would require a calibration sample with varying $3 \mathrm{D}$ orientation of dipole emitters, but the fabrication of such a sample is an immense challenge, so we decided to assess the accuracy of our method in a virtual experiment (see the Supporting Information). Using computer-generated images with added noise mimicking the conditions of real measurements, we estimated the standard deviations of the reconstructed angles $\theta$ and $\phi$. We obtained the standard deviation of the azimuthal angles $\sigma_{\theta}=5.9^{\circ}$ for polar angles $\phi>25^{\circ}$. The measurement accuracy rapidly decreased for $\phi<25^{\circ}$, which can be linked to the vanishing asymmetry of the nanorods oriented along the optical axis. The azimuthal angles are thus determined with larger error for nanoparticles aligned with the optical axis, but they also become meaningless at the same time. For polar angles, a standard deviation of $\sigma_{\phi}=7.3^{\circ}$ was estimated using the same procedure. The freely moving nanorods change not only the orientation but also their 3D position. We studied depth variations experimentally and found that the high measurement precision is maintained even with defocusing up to $500 \mathrm{~nm}$, also providing high measurement accuracy (see the Supporting Information).

Orientation Imaging of Gold Nanorods in Live-Cell Imaging. The ultimate test of the proposed method was a wide-field real-time orientation imaging of gold nanorods acting as nanoprobes in live-cell imaging. The experiment was performed with human melanoma (BLM) cells cultivated with nanorods added into the culture medium (details in the Supporting Information). The cultivated cells were observed with our $3 \mathrm{D}$ orientation imaging system (Figure 5A). In the enlarged areas shown in Figure $5 \mathrm{~B}-\mathrm{E}$, the images of nanorods that bound to the cell membrane are marked by arrows. The images were captured with a frame rate of $100 \mathrm{~Hz}$ to properly sample orientation and position changes of the nanorods. Representative time-resolved images of the nanorod bound to the cell membrane are presented in Figure 5F. Images were taken from a movie captured during the course of $9 \mathrm{~s}$. The first 500 images recorded in the course of $5 \mathrm{~s}$ were processed using the automated procedure, and the $3 \mathrm{D}$ orientation of the nanorod was reconstructed. The results are demonstrated in Figure 5G,H, showing the temporal evolution of the azimuthal angle, $\theta$, and the polar angle, $\phi$, during the interaction of the nanorod with the cell membrane. The evaluation of the nanorod movement including lateral displacement is presented in the Supporting Information. The obtained results prove that a nanorod bound to a cell membrane only slightly changes its lateral position and undergoes some nonzero in-plane rotation, while its polar angle stays close to $\phi=90^{\circ}$. However, some wobbling of the nanorod with polar angles limited to the range of $\phi>50^{\circ}$ can also be observed.

\section{CONCLUSIONS}

We have demonstrated a new optical technique allowing a single-shot wide-field $3 \mathrm{D}$ orientation imaging of elongated nanoparticles made of noble metals. Our method is based on manipulations with the angular momentum of light, namely, on SAM to OAM and reverse OAM to SAM conversion by a geometric-phase element (Q-plate). We demonstrated the method's practical applicability by the orientation imaging of randomly deposited sub-100 nm colloidal gold nanorods on a solid substrate, real-time monitoring of nanorods suspended in deionized water, and imaging of nanorods used as biological nanoprobes. The results obtained in the imaging of fixed colloidal nanorods were compared with the ground-truth values provided by SEM. The achieved accuracy of the azimuthal angle measurement was $2.5^{\circ}$. The experiments proved the capability to measure $3 \mathrm{D}$ rotational nanorod motion with a high temporal resolution, which is critical for studying dynamic phenomena occurring in practical biophotonic applications. The presented 3D orientation imaging can be extended toward the characterization of any dipolar emitter of subdiffraction size and thus can be appealing for singlemolecule fluorescence microscopy, the study of fluorescent nanoemitters, or the examination of dipole orientation in solidstate single photon emitters.

\section{ASSOCIATED CONTENT}

\section{Supporting Information}

The Supporting Information is available free of charge at https://pubs.acs.org/doi/10.1021/acs.nanolett.1c02278.

Analytical model and numerical (BEM) simulations of the proposed method, scanning electron microscopy images of gold nanorods and optical images of gold nanorods acquired using the proposed method, workflow of the numerical reconstruction of nanorod orientation from optical images, evaluation of measurement accuracy, and demonstration of the real-time optical imaging of gold nanorods (PDF)

\section{AUTHOR INFORMATION}

\section{Corresponding Author}

Petr Bouchal - Institute of Physical Engineering, Faculty of Mechanical Engineering, Brno University of Technology, 616 69 Brno, Czech Republic; Central European Institute of Technology, Brno University of Technology, 61200 Brno, Czech Republic; 이이이.org/0000-0002-6159-8099; Email: petr.bouchal@ceitec.vutbr.cz

\section{Authors}

Tomás Fordey - Department of Optics, Palacký University, 77146 Olomouc, Czech Republic

Petr Schovánek - Department of Optics, Palacký University, 77146 Olomouc, Czech Republic 
Michal Baránek - Department of Optics, Palacký University, 77146 Olomouc, Czech Republic

Zdeněk Bouchal - Department of Optics, Palacky University, 77146 Olomouc, Czech Republic

Petr Dvorák - Institute of Physical Engineering, Faculty of Mechanical Engineering, Brno University of Technology, 616 69 Brno, Czech Republic; Central European Institute of Technology, Brno University of Technology, 61200 Brno, Czech Republic; (1) orcid.org/0000-0003-3659-9249

Martin Hrton - Institute of Physical Engineering, Faculty of Mechanical Engineering, Brno University of Technology, 616 69 Brno, Czech Republic; Central European Institute of Technology, Brno University of Technology, 61200 Brno, Czech Republic

Katarína Rovenská - Institute of Physical Engineering, Faculty of Mechanical Engineering, Brno University of Technology, 61669 Brno, Czech Republic; Central European Institute of Technology, Brno University of Technology, 61200 Brno, Czech Republic

Filip Ligmajer - Institute of Physical Engineering, Faculty of Mechanical Engineering, Brno University of Technology, 616 69 Brno, Czech Republic; Central European Institute of Technology, Brno University of Technology, 61200 Brno, Czech Republic; (1) orcid.org/0000-0003-0346-4110

Radim Chmelík - Institute of Physical Engineering, Faculty of Mechanical Engineering, Brno University of Technology, 616 69 Brno, Czech Republic; Central European Institute of Technology, Brno University of Technology, 61200 Brno, Czech Republic

Tomás Sikola - Institute of Physical Engineering, Faculty of Mechanical Engineering, Brno University of Technology, 616 69 Brno, Czech Republic; Central European Institute of Technology, Brno University of Technology, 61200 Brno, Czech Republic

Complete contact information is available at:

https://pubs.acs.org/10.1021/acs.nanolett.1c02278

\section{Author Contributions}

P.B and Z.B. conceived the idea and proposed the imaging theory. M.H. performed the numerical BEM simulations. P.B., P.D., T.F., and M.B. performed the optical measurements. P.S., P.B., and M.H. were responsible for the data processing and the reconstruction of the nanorod orientation. P.D., K.R., and F.L. prepared the samples for the experiments and performed the reference SEM measurements. P.B. and Z.B wrote the

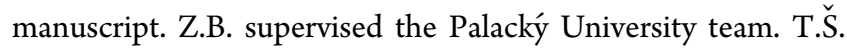
and R.C. supervised the Brno University of Technology team. All authors discussed the results and commented on the manuscript.

\section{Notes}

The authors declare no competing financial interest.

\section{ACKNOWLEDGMENTS}

This work has been supported by the Grant agency of the Czech Republic (21-01953S, 20-01673S), European Union's H2020 TWINNING project SINNCE (GA 810626), Brno University of Technology (FSI-S-20-6353, FSI-S-20-6485), and Palacký University (IGA_PrF_2021_002). We also acknowledge the Czech Nano Lab Research Infrastructure supported by MEYS CR (LM2018110).

\section{REFERENCES}

(1) Njoki, P. N.; Lim, I.-I. S.; Mott, D.; Park, H.-Y.; Khan, B.; Mishra, S.; Sujakumar, R.; Luo, J.; Zhong, C.-J. Size Correlation of Optical and Spectroscopic Properties for Gold Nanoparticles. J. Phys. Chem. C 2007, 111 (40), 14664-14669.

(2) González, A. L.; Noguez, C.; Beránek, J.; Barnard, A. S. Size, Shape, Stability, and Color of Plasmonic Silver Nanoparticles. J. Phys. Chem. C 2014, 118 (17), 9128-9136.

(3) Stender, A. S.; Marchuk, K.; Liu, C.; Sander, S.; Meyer, M. W.; Smith, E. A.; Neupane, B.; Wang, G.; Li, J.; Cheng, J.-X.; Huang, B.; Fang, N. Single Cell Optical Imaging and Spectroscopy. Chem. Rev. 2013, 113 (4), 2469-2527.

(4) Saha, K.; Agasti, S. S.; Kim, C.; Li, X.; Rotello, V. M. Gold Nanoparticles in Chemical and Biological Sensing. Chem. Rev. 2012, 112 (5), 2739-2779.

(5) Sönnichsen, C.; Franzl, T.; Wilk, T.; von Plessen, G.; Feldmann, J.; Wilson, O.; Mulvaney, P. Drastic Reduction of Plasmon Damping in Gold Nanorods. Phys. Rev. Lett. 2002, 88 (7), 077402.

(6) Nikoobakht, B.; El-Sayed, M. A. Preparation and Growth Mechanism of Gold Nanorods (NRs) Using Seed-Mediated Growth Method. Chem. Mater. 2003, 15 (10), 1957-1962.

(7) Chang, W.-S.; Ha, J. W.; Slaughter, L. S.; Link, S. Plasmonic Nanorod Absorbers as Orientation Sensors. Proc. Natl. Acad. Sci. U. S. A. 2010, 107 (7), 2781-2786.

(8) Hamon, C.; Novikov, S. M.; Scarabelli, L.; Solís, D. M.; Altantzis, T.; Bals, S.; Taboada, J. M.; Obelleiro, F.; Liz-Marzán, L. M. Collective Plasmonic Properties in Few-Layer Gold Nanorod Supercrystals. ACS Photonics 2015, 2 (10), 1482-1488.

(9) Lim, S.; Lee, M.; Kang, S.; Dey, J.; Umar, A.; Lee, S.; Choi, S. Individually Silica-Embedded Gold Nanorod Superlattice for High Thermal and Solvent Stability and Recyclable SERS Application. Adv. Mater. Interfaces 2019, 6 (21), 1900986.

(10) Rožič, B.; Fresnais, J.; Molinaro, C.; Calixte, J.; Umadevi, S.; Lau-Truong, S.; Felidj, N.; Kraus, T.; Charra, F.; Dupuis, V.; Hegmann, T.; Fiorini-Debuisschert, C.; Gallas, B.; Lacaze, E. Oriented Gold Nanorods and Gold Nanorod Chains within Smectic Liquid Crystal Topological Defects. ACS Nano 2017, 11 (7), 67286738.

(11) Ohashi, Y.; Ranjan, B.; Saito, Y.; Umakoshi, T.; Verma, P. Tapered Arrangement of Metallic Nanorod Chains for Magnified Plasmonic Nanoimaging. Sci. Rep. 2019, 9 (1), 2656.

(12) Harms, G. S.; Sonnleitner, M.; Schütz, G. J.; Gruber, H. J.; Schmidt, T. Single-Molecule Anisotropy Imaging. Biophys. J. 1999, 77 (5), 2864-2870.

(13) Huang, Y.; Kim, D.-H. Dark-Field Microscopy Studies of Polarization-Dependent Plasmonic Resonance of Single Gold Nanorods: Rainbow Nanoparticles. Nanoscale 2011, 3 (8), 3228.

(14) Ming, T.; Zhao, L.; Yang, Z.; Chen, H.; Sun, L.; Wang, J.; Yan, C. Strong Polarization Dependence of Plasmon-Enhanced Fluorescence on Single Gold Nanorods. Nano Lett. 2009, 9 (11), 38963903.

(15) Sönnichsen, C.; Alivisatos, A. P. Gold Nanorods as Novel Nonbleaching Plasmon-Based Orientation Sensors for Polarized Single-Particle Microscopy. Nano Lett. 2005, 5 (2), 301-304.

(16) He, J.; Zheng, W.; Ligmajer, F.; Chan, C.-F.; Bao, Z.; Wong, K.L.; Chen, X.; Hao, J.; Dai, J.; Yu, S.-F.; Lei, D. Y. Plasmonic Enhancement and Polarization Dependence of Nonlinear Upconversion Emissions from Single Gold Nanorod@SiO2@CaF2:Yb3+,Er3+ Hybrid Core-Shell-Satellite Nanostructures. Light: Sci. Appl. 2017, 6 (5), e16217-e16217.

(17) Failla, A. V.; Qian, H.; Qian, H.; Hartschuh, A.; Meixner, A. J. Orientational Imaging of Subwavelength Au Particles with Higher Order Laser Modes. Nano Lett. 2006, 6 (7), 1374-1378.

(18) Xiao, L.; Qiao, Y.; He, Y.; Yeung, E. S. Three Dimensional Orientational Imaging of Nanoparticles with Darkfield Microscopy. Anal. Chem. 2010, 82 (12), 5268-5274.

(19) Lee, J.; Ha, J. W. Defocused Dark-Field Orientation Imaging of Single Gold Microrods on Synthetic Membranes. Phys. Chem. Chem. Phys. 2017, 19 (36), 24453-24457. 
(20) Li, T.; Li, Q.; Xu, Y.; Chen, X.-J.; Dai, Q.-F.; Liu, H.; Lan, S.; Tie, S.; Wu, L.-J. Three-Dimensional Orientation Sensors by Defocused Imaging of Gold Nanorods through an Ordinary WideField Microscope. ACS Nano 2012, 6 (2), 1268-1277.

(21) Wang, G.; Sun, W.; Luo, Y.; Fang, N. Resolving Rotational Motions of Nano-Objects in Engineered Environments and Live Cells with Gold Nanorods and Differential Interference Contrast Microscopy. J. Am. Chem. Soc. 2010, 132 (46), 16417-16422.

(22) Gu, Y.; Sun, W.; Wang, G.; Jeftinija, K.; Jeftinija, S.; Fang, N. Rotational Dynamics of Cargos at Pauses during Axonal Transport. Nat. Commun. 2012, 3 (1), 1030.

(23) Zhao, F.; Chen, K.; Dong, B.; Yang, K.; Gu, Y.; Fang, N. Localization Accuracy of Gold Nanoparticles in Single Particle Orientation and Rotational Tracking. Opt. Express 2017, 25 (9), 9860.

(24) Chen, K.; Gu, Y.; Sun, W.; Dong, B.; Wang, G.; Fan, X.; Xia, T.; Fang, N. Characteristic Rotational Behaviors of Rod-Shaped Cargo Revealed by Automated Five-Dimensional Single Particle Tracking. Nat. Commun. 2017, 8 (1), 887.

(25) Overvig, A. C.; Shrestha, S.; Malek, S. C.; Lu, M.; Stein, A.; Zheng, C.; Yu, N. Dielectric Metasurfaces for Complete and Independent Control of the Optical Amplitude and Phase. Light: Sci. Appl. 2019, 8 (1), 92.

(26) Wang, X.; Nie, Z.; Liang, Y.; Wang, J.; Li, T.; Jia, B. Recent Advances on Optical Vortex Generation. Nanophotonics 2018, 7 (9), $1533-1556$.

(27) Arbabi, A.; Horie, Y.; Bagheri, M.; Faraon, A. Dielectric Metasurfaces for Complete Control of Phase and Polarization with Subwavelength Spatial Resolution and High Transmission. Nat. Nanotechnol. 2015, 10 (11), 937-943.

(28) Zhao, R.; Huang, L.; Tang, C.; Li, J.; Li, X.; Wang, Y.; Zentgraf, T. Nanoscale Polarization Manipulation and Encryption Based on Dielectric Metasurfaces. Adv. Opt. Mater. 2018, 6 (19), 1800490.

(29) Bouchard, F.; De Leon, I.; Schulz, S. A.; Upham, J.; Karimi, E.; Boyd, R. W. Optical Spin-to-Orbital Angular Momentum Conversion in Ultra-Thin Metasurfaces with Arbitrary Topological Charges. Appl. Phys. Lett. 2014, 105 (10), 101905.

(30) Devlin, R. C.; Ambrosio, A.; Rubin, N. A.; Mueller, J. P. B.; Capasso, F. Arbitrary Spin-to-Orbital Angular Momentum Conversion of Light. Science (Washington, DC, U. S.) 2017, 358 (6365), 896-901.

(31) Marrucci, L.; Manzo, C.; Paparo, D. Optical Spin-to-Orbital Angular Momentum Conversion in Inhomogeneous Anisotropic Media. Phys. Rev. Lett. 2006, 96 (16), 163905.

(32) Hsiao, H.-H.; Chu, C. H.; Tsai, D. P. Fundamentals and Applications of Metasurfaces. Small Methods 2017, 1 (4), 1600064.

(33) Kim, J.; Li, Y.; Miskiewicz, M. N.; Oh, C.; Kudenov, M. W.; Escuti, M. J. Fabrication of Ideal Geometric-Phase Holograms with Arbitrary Wavefronts. Optica 2015, 2 (11), 958.

(34) Greengard, A.; Schechner, Y. Y.; Piestun, R. Depth from Diffracted Rotation. Opt. Lett. 2006, 31 (2), 181.

(35) Park, Y.; Shin, S.; Jin, H.; Park, J.; Hong, Y.; Choi, J.; Jung, B.; Song, H.; Seo, D. Single-Molecule Rotation for EGFR Conformational Dynamics in Live Cells. J. Am. Chem. Soc. 2018, 140 (45), 15161-15165.

(36) Rosenberg, S. A.; Quinlan, M. E.; Forkey, J. N.; Goldman, Y. E. Rotational Motions of Macro- Molecules by Single-Molecule Fluorescence Microscopy. Acc. Chem. Res. 2005, 38 (7), 583-593.

(37) Shao, L.; Yang, Z.-J.; Andrén, D.; Johansson, P.; Käll, M. Gold Nanorod Rotary Motors Driven by Resonant Light Scattering. ACS Nano 2015, 9 (12), 12542-12551.

(38) Allen, L.; Beijersbergen, W. M.; Spreeuw, R. J. C.; Woerdman, J. P. Orbital Angular Momentum of Light and the Transformation of Laguerre-Gaussian Laser Modes. Phys. Rev. A: At., Mol., Opt. Phys. 1992, 45 (11), 8185-8189.

(39) Shen, Y.; Wang, X.; Xie, Z.; Min, C.; Fu, X.; Liu, Q.; Gong, M.; Yuan, X. Optical Vortices 30 Years on: OAM Manipulation from Topological Charge to Multiple Singularities. Light: Sci. Appl. 2019, 8 (1), 90.
(40) Rubinsztein-Dunlop, H.; Forbes, A.; Berry, M. V.; Dennis, M. R.; Andrews, D. L.; Mansuripur, M.; Denz, C.; Alpmann, C.; Banzer, P.; Bauer, T.; Karimi, E.; Marrucci, L.; Padgett, M.; Ritsch-Marte, M.; Litchinitser, N. M.; Bigelow, N. P.; Rosales-Guzmán, C.; Belmonte, A.; Torres, J. P.; Neely, T. W.; Baker, M.; Gordon, R.; Stilgoe, A. B.; Romero, J.; White, A. G.; Fickler, R.; Willner, A. E.; Xie, G.; McMorran, B.; Weiner, A. M. Roadmap on Structured Light. J. Opt. 2017, 19 (1), 013001.

(41) Matsuo, S. Matrix Calculus for Axially Symmetric Polarized Beam. Opt. Express 2011, 19 (13), 12815.

(42) Pavani, S. R. P.; Thompson, M. A.; Biteen, J. S.; Lord, S. J.; Liu, N.; Twieg, R. J.; Piestun, R.; Moerner, W. E. Three-Dimensional, Single-Molecule Fluorescence Imaging beyond the Diffraction Limit by Using a Double-Helix Point Spread Function. Proc. Natl. Acad. Sci. U. S. A. 2009, 106 (9), 2995-2999.

(43) Lu, X.; Wu, J.; Zhu, Q.; Zhao, J.; Wang, Q.; Zhan, L.; Ni, W. Circular Dichroism from Single Plasmonic Nanostructures with Extrinsic Chirality. Nanoscale 2014, 6 (23), 14244-14253.

(44) Yu, N.; Genevet, P.; Kats, M. A.; Aieta, F.; Tetienne, J. P.; Capasso, F.; Gaburro, Z. Light Propagation with Phase Discontinuities: Generalized Laws of Reflection and Refraction. Science (Washington, DC, U. S.) 2011, 334 (6054), 333-337.

(45) Zhu, A. Y.; Chen, W. T.; Zaidi, A.; Huang, Y.-W.; Khorasaninejad, M.; Sanjeev, V.; Qiu, C.-W.; Capasso, F. Giant Intrinsic Chiro-Optical Activity in Planar Dielectric Nanostructures. Light: Sci. Appl. 2018, 7 (2), 17158-17158. 